\title{
Research on Novel 3D Modelling Methodology for Animation Design based on Computer Graphics Theory and Mathematical Analysis with Kinect Implementation
}

\author{
Honglin Huang \\ Guangzhou International Economics College, Guangdong 510540, China \\ honglinhuang1@126.com
}

\begin{abstract}
At present, the $3 D$ object model technique is being applied in the simulation, virtual reality, 3D film industry and culture protection, and many other fields which can be widely applied. However, the existing $3 D$ reconstruction technology is based on the complex and often expensive sensors. Therefore, we conduct research on computer graphics theory and mathematical modelling based $3 D$ reconstruction methodology with Kinect. We firstly introduce the basic concepts and application of Kinect system which serves as the preliminary of our research. Then, we conduct mathematical modelling for our proposed system with theoretical analysis. Later, experiment and numerical simulation is conducted for verifying the robustness and effectiveness of our proposed approach. The visualized and numerical simulation result indicates that our method outperforms and holds better accuracy compared with other popular adopted algorithms. In the future, we plan to combine more optimization techniques to polish and modify our approach.
\end{abstract}

Keywords: 3D Modelling and Reconstruction, Animation Design, Computer Graphics Theory, Mathematical Analysis; Image Processing

\section{Introduction}

3D modelling and reconstruction as one of the most important research topics in the community of computer graphics, mathematical modelling and pattern recognition is becoming more and more fascinating to researchers [1]. Animation industry as a sunrise industry in the 21 st century, in our country has developed rapidly in recent years, however, as the forefront of the whole animation industry and the development direction of 3D animation encountered many problems in its development process. Among them, the modelling of long production period and high cost of design, such as lack of innovative become outstanding problems. In recent years, experts use the evolutionary computation method for the study of a large number of innovative designs. As the foundation of the animation design, computer graphics theory has been applied to a great deal of applications which could be categorized into the following parts. (1) Rendering applications. In [2], Holly et al. conduct research on computer graphics material model acquisition. They consider a cheap system for materials design model of computer graphics in the application of animation, games and conceptual design. In these applications useful system must be able to model trained rich, in the form of representation. (2) Data analysis and classification. In [3], Ken et al. introduce the basic concepts and principles of scattered data interpolation for the applications of computer graphics theory and related areas. In their review, they can be used to model a surface from a set of sparse samples, to reconstruct a BRDF from a set of measurements, or to interpolate motion capture data. (3) Visualization and virtual reality. In [4], Qi's research group focuses on the research of designing computer graphics and visual communication system to find out solution for current systems. They point out that computer graphics 
technology pay attention to the operation of the computer technology, more focused on the design of the performance art, especially pay attention to the cultivation of innovative consciousness. (4) Machine learning. In [5], Upulee et al. conducted research on predicting metamorphic relations for testing scientific software based on computer graphics. The find out that metamorphic testing is a method for automating the testing process for programs without test oracles. This technique operates by checking whether the program behaves according to a certain set of properties called metamorphic relations. (5) Mathematical modelling and analysis. In [6], Jan et al. get advancement on interactive simulation of rigid body. They combine the computer graphics theory with mathematical modelling technique to solve the current problem.

At present, the 3D object model in the simulation, virtual reality, 3D film industry and culture protection, and many other fields, can be widely applied. However, the existing $3 \mathrm{D}$ reconstruction technology based on the complex and often expensive sensors, such as structure light or laser rangefinder camera. If the 3D reconstruction can be cheaper, make the 3D object model as readily available, such as photographs and video object model can be used in more fields, such as e-commerce platform and online shopping sites. Therefore, how to quickly and cheaply finalize object modeling is an important goal of computer graphics. Kinect device sensors, may say is a revolutionary change. It was Microsoft's development host Xbox360 surrounding peripherals, mainly used for human-computer interaction, real-time but there are also some literature reports to be used in 3D reconstruction. We firstly need to human body modeling. Then use the image feature points to realize the local registration of adjacent frames, and carry on global optimization. The system uses the local registration and repeated iteration to get the global optimization model of the human body. The system need to human body modeling, does not apply to general object reconstruction, because of general object image, it is difficult to extract reliable matching characteristics. In addition, the iterative registration strategy will be to a certain extent influence the time performance of the algorithm. The advantage of Kinect for 3D reconstruction of computer graphics is separated into two parts: (1) Kinect can quickly get the depth of the scene information. (2) Kinect is a kind of active sensor it is not affected by the environment of the visible spectrum interference.

In this paper, we conduct research on novel 3D modelling methodology for animation design based on computer graphics theory and mathematical analysis with Kinect implementation. We firstly introduce the basic concepts and application of Kinect system which serves as the preliminary of our research. Then, we conduct mathematical modelling for our system with theoretical analysis. Later, experiment and numerical simulation is conducted for verifying the robustness and effectiveness of our proposed approach. As the final conclusion, we compare our method with other approaches and set up the prospect.

\section{The Introduction to Kinect System and Applications}

\subsection{The Kinect Introduction}

The object of this article reconstruction system mainly includes three devices: a device sensor, a rotating platform and a computer equipped with GPU. Rotating platform by a step motor connected to the computer. The rotation of the stepper motor control rotating platform, implement platform on the rotary motion of the object [7-11]. Will to rebuild the object under central turntable, can start system, object reconstruction in system first drive turntable rotation, and then using the device sensor parallax graphs are taken at regular intervals. By collecting the parallax diagram, system online calculated RGB camera coordinates of 3D point cloud, and the real-time local registration with adjacent point cloud. At the end of the rotation and acquisition, the sequences of 3D point cloud: $\left(X_{0}, X_{1}, \ldots, \mathrm{X}_{n-1}\right)$. Finally, the combination of point cloud and filter, get the $3 \mathrm{~d}$ model 
object. By the principle of the reconstruction of the above, our object reconstruction method mainly has the following several steps: (1) Calibration device sensors. Related parameters calibration content includes: the infrared camera, color cameras within the parameters of the rigid body transformation of matrix and the infrared camera and color camera. For point $\mathrm{P}$ in the space, picked by device got its parallax $\mathrm{d}$, under the infrared camera coordinate system can be made of the infrared camera calibration parameter calculation point $\mathrm{P}$ in the 3D coordinates of infrared camera coordinate system. Then, according to the rotation of the color video camera and infrared cameras within the relationship between translation and color video camera parameter matrix, the calculation point $\mathrm{P}$ in color video camera coordinates of $3 \mathrm{D}$ coordinates and their corresponding image coordinates. (2) Calibration device and the changing relationship. In a fixed device sensors and the relative position of turntable, needs to be done on the relationship between the device and the turntable calibration. As a result of the 3D model generation depends on the result of the point cloud registration, and the point cloud registration error will cause the failure of reconstruction. Therefore, we use rough calibration value to guarantee registration results do not appear large error, in order to enhance the system robustness. (3) Point cloud to obtain, registration and optimization. This step to rebuilding of objects in the middle of the turntable, the main process is divided into two parts of online and offline calculation.

\subsection{The Kinect Calibration and Pre-Processing}

Reconstruction after the start, the system first real-time access point cloud, and calculate the relative relations between adjacent point clouds of group $\mathrm{c}$. For point $\mathrm{P}$ in the space, picked by device got its parallax d under the infrared camera coordinate system. Therefore, the coordinate transformation is expressed as the formula 1.

$$
\left(\begin{array}{c}
x_{R G B} \\
y_{R G B} \\
z_{R G B}
\end{array}\right)=R_{R G B}-I R\left(\begin{array}{c}
x_{I R} \\
y_{I R} \\
z_{I R}
\end{array}\right)+t_{R G B-T R}
$$

Monocular camera pose estimation problem is according to the camera in the image sequence of discrete sampling point to be treated, through the establishment of feature matching between images, and the constraint relationship between the camera movement and the camera motion parameters calculation. Usually the camera motion can be regarded as rigid body motion, as a result, the camera's position at any time during a two parameter can move through a rotating $\mathrm{R}$ and $\mathrm{t}$. The incremental parameterized model of feature point is shown in the Figure 1. The mathematical expression is shown in the formula 2 .

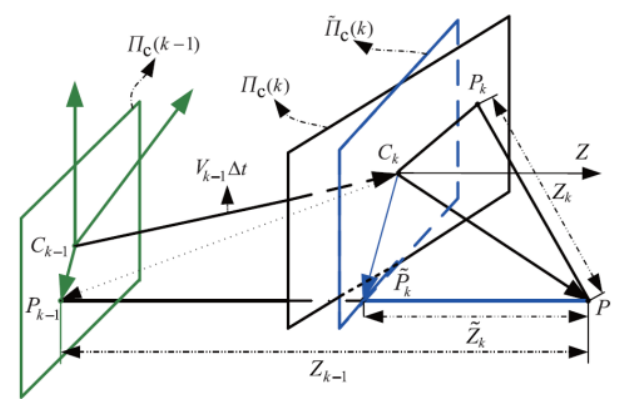

Figure 1.The Incremental Parameterized Model 


$$
Z_{i}(k)=\frac{1}{K(k)}\left(\left(Z_{i}-\left.V_{c}\right|_{3 \Delta t}\right)^{2}+\left\|\frac{Z_{i}}{f} I_{i}-V_{c} \Delta t\right\|\right)^{1 / 2}
$$

System observability is an important issue in the field of state estimation which provides the accuracy of the system state estimation with an intuitive way of understanding. For a monocular camera since this kind of strongly nonlinear model, the motion estimation observability analysis directly is very difficult. Therefore, in order to effectively observability analysis, need to convert it to time-varying linear system. Similarly, based on the Euclidean observability analysis of parametric model which can find their corresponding linear model of Taylor expansion is significant sex cannot be guaranteed. The expression is shown blow.

$$
A_{k}=\left.\frac{\partial f(X)}{\partial X}\right|_{X=X_{k-1}}, C_{k}=\left.\frac{\partial g(X)}{\partial X}\right|_{X=X_{k-1}}
$$

\section{Our Proposed Method}

\subsection{The Principles of Cellular Genetic Algorithm}

Cellular genetic algorithm (CGA) is the cellular automata and the combination of genetic algorithm, the algorithm using cellular automaton evolution rules instead of the cross mechanism in the genetic algorithm, its core lies in the genetic operation is limited to the individual's neighborhood, repeated local interactions, ultimately achieve the goal of search the global optimal solution [12-15]. For cellular genetic algorithm (CGA) encoding is not flexible and innovative design unified evaluation criterion, this paper adopts flexible tree structure coding and introduce the man-machine interactive way, an improved cellular genetic algorithm is proposed. Cellular genetic algorithm commonly used in encoding and standard the same fixed-length binary coded genetic algorithm (GA), although this encoding method is simple in technology, but is not flexible, and need to encode the domain to the problem domain mapping. Length and content changes to facilitate the generation of regular expressions, this paper adopted a more flexible way of coding based on tree structure. The probability of the whole region $\mathrm{R}$ to belong to class $\mathrm{c}$ can be computed as the joint probability over all its pixels. Assuming the pixels to have independent labels, the joint probability is:

$$
E(\pi)=\sum_{i} E\left(c_{i} \mid R_{i}\right)=-\sum_{i} \sum_{x \in R_{i}} \log p\left(c_{1} \mid f_{c}\right)
$$

We started from the initial seeds the seeds of random consider community if possible instance of construction and better conform to the definition of the energy of the input image. If so, the seed is updated, we continue to explore new near one, according to the following algorithm which is denote to be the modelling procedure:

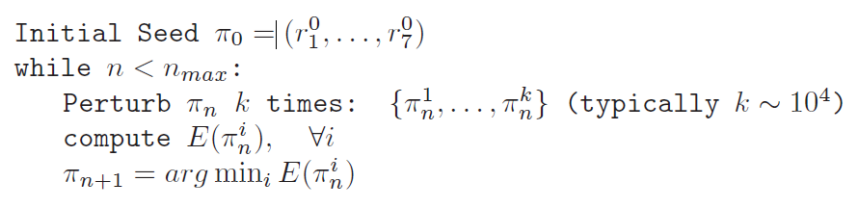

Anime modelling design is a design activity is given priority to with people's subjective orientation, because in the process of innovation design involves a lot of hidden indicators, such as modelling beautiful, simulation modelling, modelling originality degrees. Among them, the cell operation is to point to in the center of the individual within the neighborhood of the operation of the genetic operators, namely 
selection centers of cellular neighbor two individuals perform crossover operation, to generate new individuals crossover mutation of the individual, as shown in the process, and, among them, the central element cell type uses the L5 neighborhood, as shown in Figure surrounded by thick line area; Cellular genetic algorithm update strategy refers to the interaction between local neighborhood of a planning mechanism, according to the different update strategy, CGA is divided into synchronous asynchronous cellular genetic algorithm (GA) cell and the genetic algorithm. In the use of cellular genetic algorithm for anime modelling design process, as the main indicator of success for a cartoon modelling, recessive indexes with clear functions are hard. Therefore cellular genetic algorithm is presented in the paper, on the basis of introducing the man-machine interactive way, through expert knowledge to determine the value of the individual to replace the traditional fitness function is calculated. The standard is shown below.

$$
\text { Standarad }=\alpha \times \text { Bea }+\beta \times \text { Sim }+\gamma \times \text { Ori }
$$

In addition, design experts can also be set to reduce the modelling of the dominant index score of labor, reduce fatigue grading. Before the shape deformation, for example, by setting the modelling of the head and the proportion of the modelling of the proportion of the body or facial features, the system shows only proportion standard modelling to expert scoring which reduces the number of expert scoring.

\subsection{The Image Depth Estimation Algorithm}

So far, the two methods can handle a distortion. The first is the special application of each camera calibration and estimate a distortion model, can be used to correct the followup image produced by a camera. This difference from the reconstruction of the deformation of simple method of input data is not executed distortion estimation and camera position. In addition, can be transformed into rigid camera positioning components and a range of static deformation components allows us to greatly reduce the number of estimated parameters than the average non-rigid deformation. This compact parameterized make extremely efficient synchronous positioning and calibration. Then the measurement metrics is formulated to the expression 6 as the point-plane length. The Figure 2 visualizes the step.

$$
E_{a}=(T, C)=\sum_{i, j} \sum_{k i, j}\left((p-q)^{T} n_{p}\right)+\lambda E_{r}(C)
$$
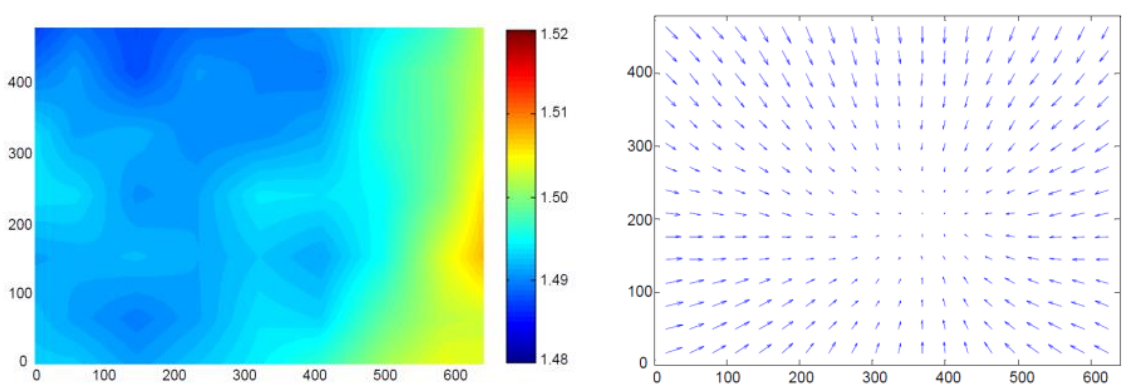

\section{Figure 2.The Visualized Flow-chat of the Depth Estimation Procedure}

If our goal is to locate the word itself, nothing can stop a degradation of the solution and the scene is a single point in space to collapse. In this solution, the calibration function mapping the measured quantity to a certain extent, and arrange the camera poses and these points are all aligned in the world. Thus, spline is a skeleton based 3D tubular physical representation. It entities are defined with the spline area of skeleton (center) and thickness (radius), accurate representation. This provides the interactive modeling is convenient. On the one hand, the ball b-spline to tubular object modeling, simply enter the corresponding control points of $3 \mathrm{~d}$ coordinate and control the radius, according to the 
given formula to calculate the entity at a point on the surface, in turn connected to the grid, it can realize the establishment of the model; On the other hand, to control the model, the operation such as deformation which can be directly by changing the control point position and radius. Aligned term minimize to zero value in the solution, so it is necessary to adjust some form clearly. The approximation function is shown below.

$$
\begin{aligned}
& T_{i} \approx\left(\begin{array}{cccc}
1 & -\gamma_{i} & \beta_{i} & \alpha_{i} \\
\gamma_{i} & 1 & -\alpha_{i} & b_{i} \\
-\beta_{i} & \alpha_{i} & 1 & c_{i} \\
0 & 0 & 0 & 1
\end{array}\right) \times\left(\begin{array}{cccc}
1 & -\gamma_{i} & \beta_{i} & \alpha_{i} \\
\gamma_{i} & 1 & -\alpha_{i} & b_{i} \\
-\beta_{i} & \alpha_{i} & 1 & c_{i} \\
0 & 0 & 0 & 1
\end{array}\right)^{\mathrm{T}} \cdot T_{i}^{k} \\
& \forall(i, j) \in O_{m}: w_{m} \cdot X_{i}>w_{m} \cdot X_{j} \\
& \forall(i, j) \in S_{m}: w_{m} \cdot X_{i}>w_{m} \cdot X_{j}
\end{aligned}
$$

Set of 3D modeling of a key challenge is to identify related components are presented to the user. Unless modeling the task ahead of time is fixed an assembly-based modeling tools have to use a lot of and different components of a database. Interface so you need to provide effective mechanism to determine the most relevant components available in any phase of the modeling process. The formulation is shown blow.

$$
\text { minimize } \frac{1}{2}\left\|w_{m}\right\|_{2}^{2}+\alpha \sum_{i, j}\left(L\left(\gamma_{i j}\right)+L\left(\zeta_{i j}\right)\right)
$$

We establish probability model in order to recommend components which can increase a given 3D model. The categories and the parts within each category are ordered based on their compatibility with the current shape, as computed by the probabilistic model. The order of the categories and parts is automatically updated when the composition of the assembled shape changes. Probability model is in a group of segmentation and the shape of the label. Each adjacent geometric shape is composed of existence, style, symmetry, from each category and the number of components, as described below. These properties are represented as random variables. The clusters are shown in the Figure 3.

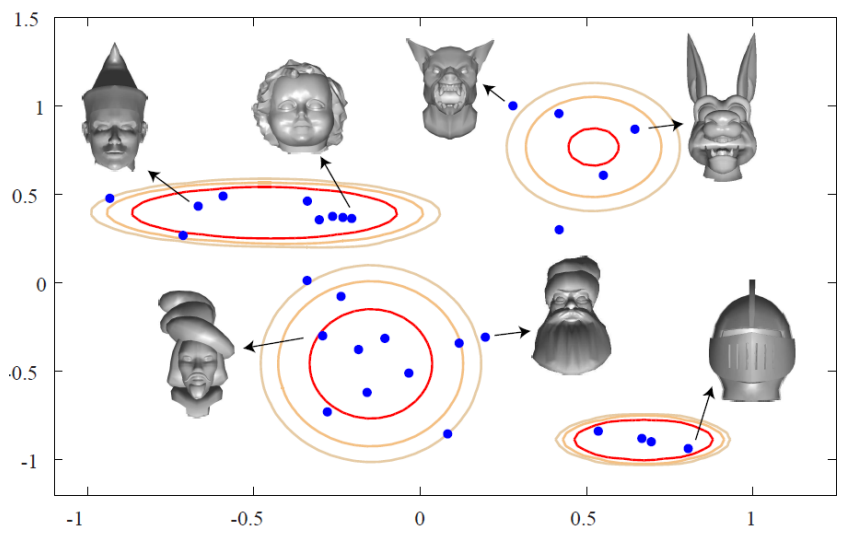

Figure 3.The Visualized Clusters Expression

\subsection{The Combination with Poser Model}

So far, the two methods can handle a distortion. The first is the special application of each camera calibration. Skeleton by key points and end points is according to the topology of the joints with the attachment. Because of the different of body shape, joint position is also different. Key points of calculation using the method of center, which is to select parent joint subsidiary and affiliate section of the center of the boundary intersection part of the current joint as the center of the current joint shaft. If the camera parameters are known and then based on the change of feature point image coordinates, to 
restore the feature point position in world coordinates, it is also the basic principle of parallax method. Based on feature points for repeated observation, can with the help of the parallax differences, get the monocular camera movement scale information which is selected to be compared with traditional XYZ Euclidean parameterized model of incremental parameterized model on observability and model dimension has obvious advantages, based on the filtering of the observable model estimation precision can be improved. For the movement speed of incremental model, the repetition of the observed feature points to establish the parameterized model, can effectively solve the widespread movement in monocular visual dimension fuzzy problems, we think this is due to the established model is completely observable results. In addition, with the introduction of characteristics of depth information readily available in the scene to access system, to perfect scene depth of the feature points incremental model, can get more accurate camera motion parameters estimation results. This method, the need for the observed scene characteristic parameterized modeling, and augmented state equation to the navigation system. Commonly used parametric model is Euclidean XYZ coordinate parameterized model, the model of feature points directly to the world coordinate system coordinates augmented to state variables, so as to realize the scene feature location estimation. When the scene feature optical center far distance from the camera or parallax angle is small, the model will introduce greater error. In describing human movement model, each body paragraph abstract for simple rigid geometric entities, joint abstraction as a sphere, with the joints between the relative position and attitude change describe human movement. The current joint position or attitude change, attached to the body segment joints and its joint changes, produce attached to the skeleton of the rigid motion of the skin. We could visually show the process in the Figure 4.

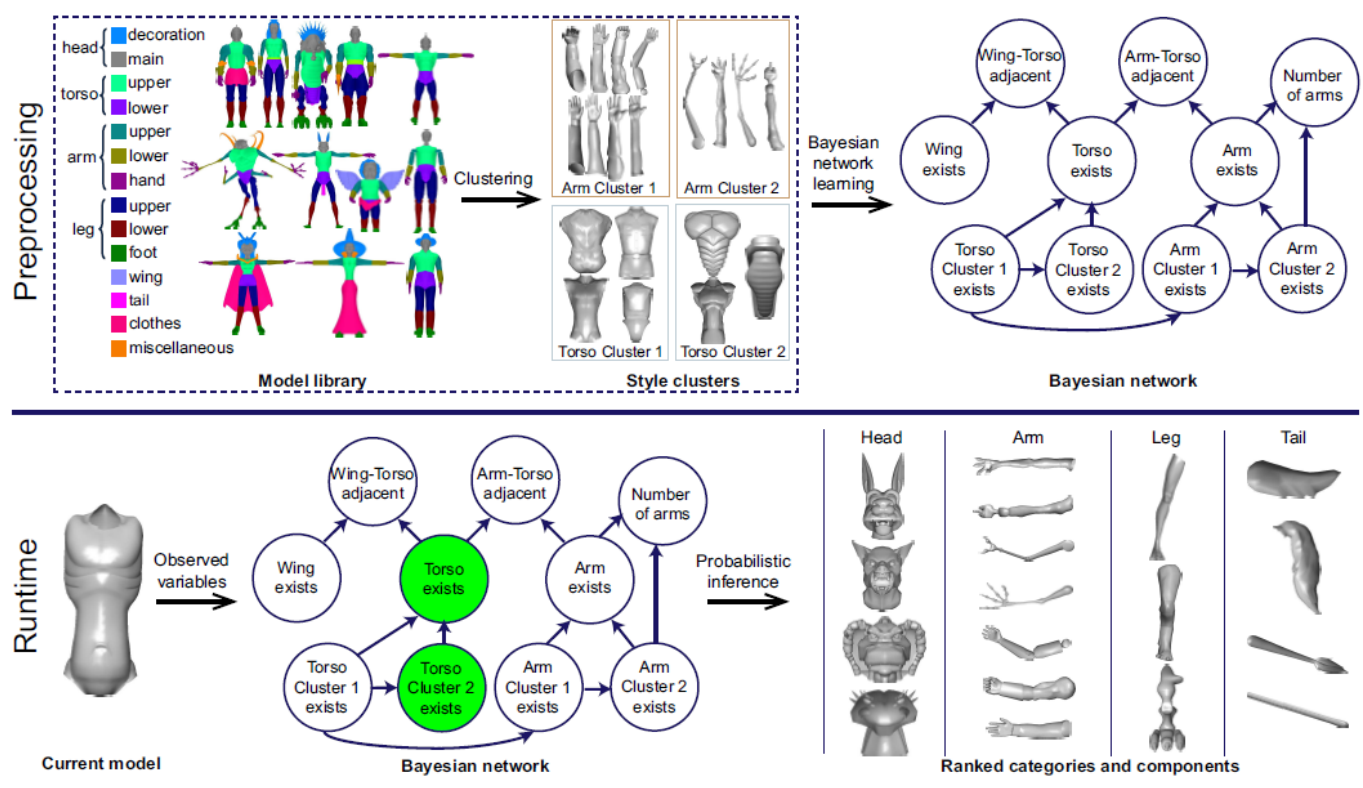

Figure 4.The Explanation of the Combined Model

\section{The Experiment and Simulation Result}

\subsection{Experimental Set up and Initialization}

Our presentation components probability model is compared to the use of two methods. The first option is a static sequence classification and parts. Before ordering is based on the probability of the category and style in the training data clustering. So more frequently 
use category and geometric style appears in the first place. The hardware environment for the experiment is standard. The result will be shown in the next section.

\subsection{The Visual Result for the Experiment}

To test the effectiveness and robustness of the proposed methodology, we take the experimental simulation for our proposed framework. The following Figure 5-8 shows the visual result of the experiment. We could conclude that the result is satisfactory.

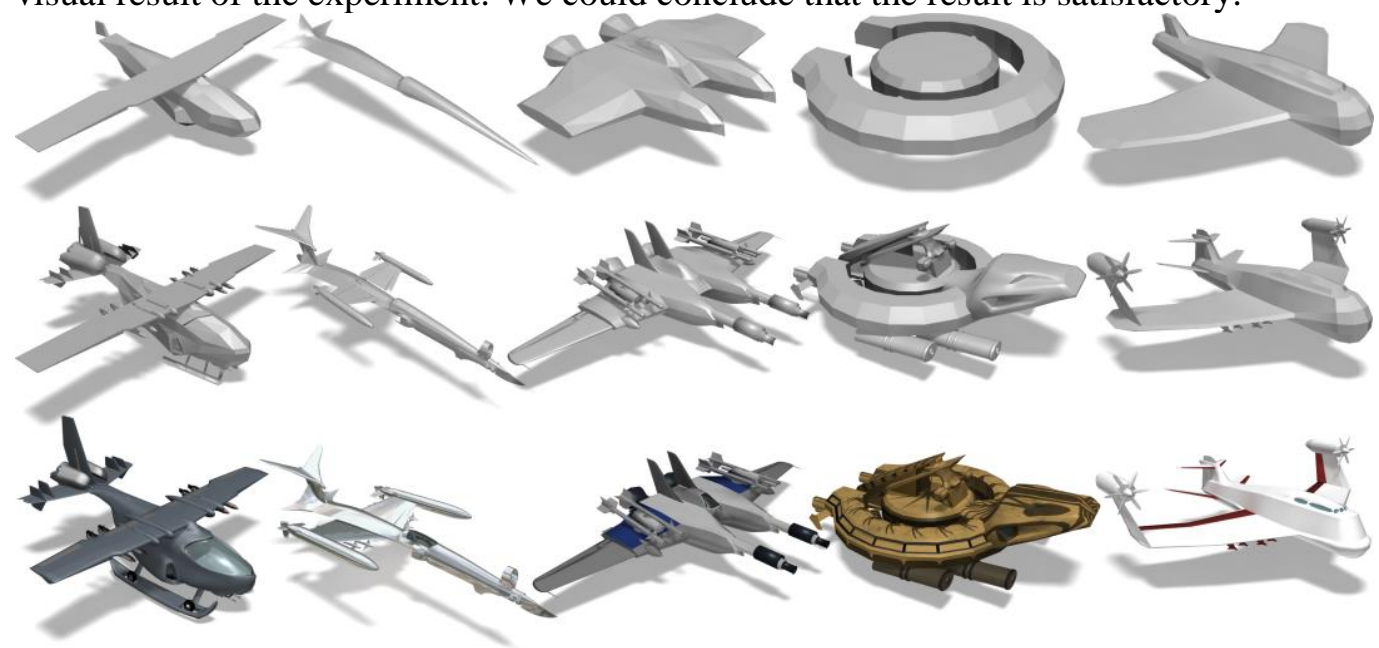

Figure 5. The Experiment Set One for Our Methodology

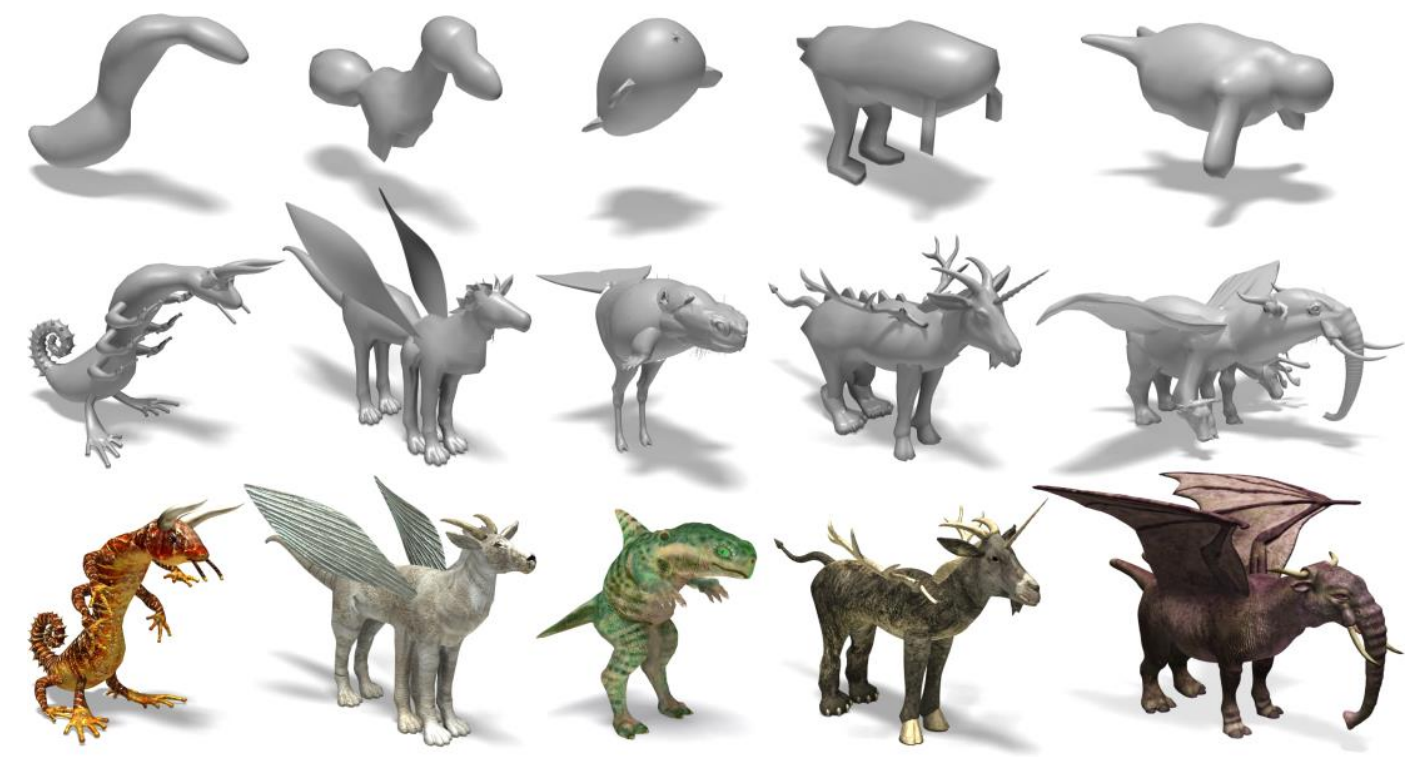

Figure 6.The Experiment Set Two for Our Methodology 

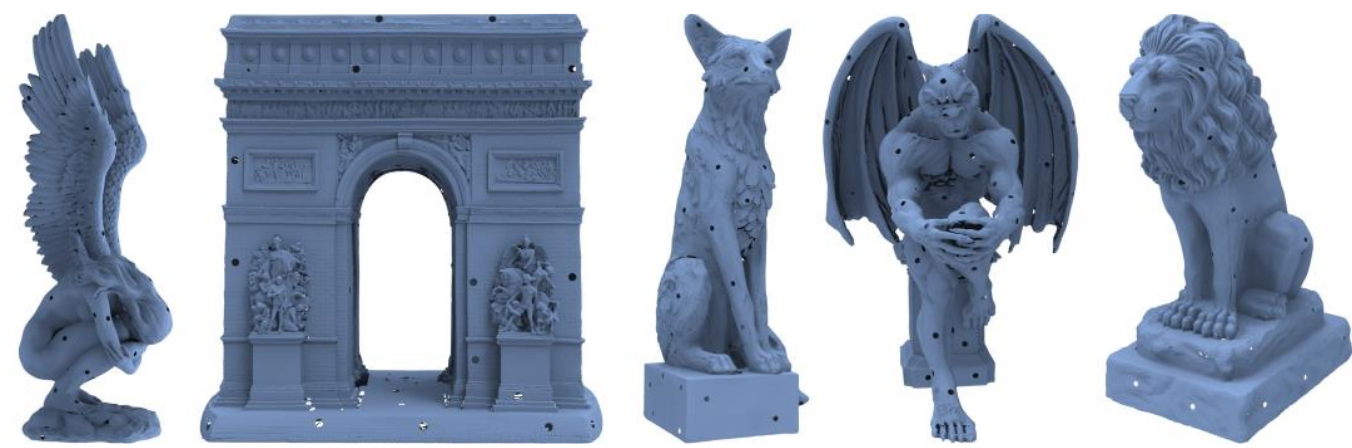

Figure 7. The Experiment Set Three for Our Methodology

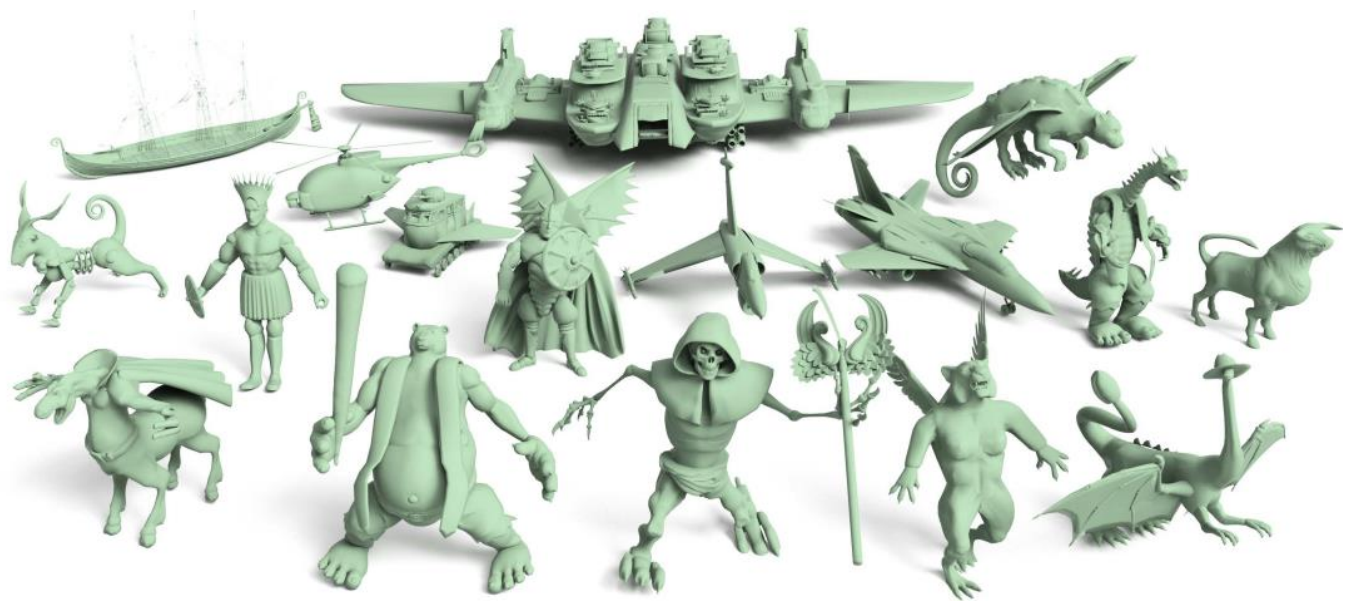

Figure 8.The Experiment Set Four for Our Methodology

\subsection{The Numerical Result for the Experiment}

To test the feasibility and effectiveness of our method compared with other state-offthe-art algorithms we undertake the comparison experiment to analysis the result of the experiment numerically and more visually. The Figure 9 shows the result.
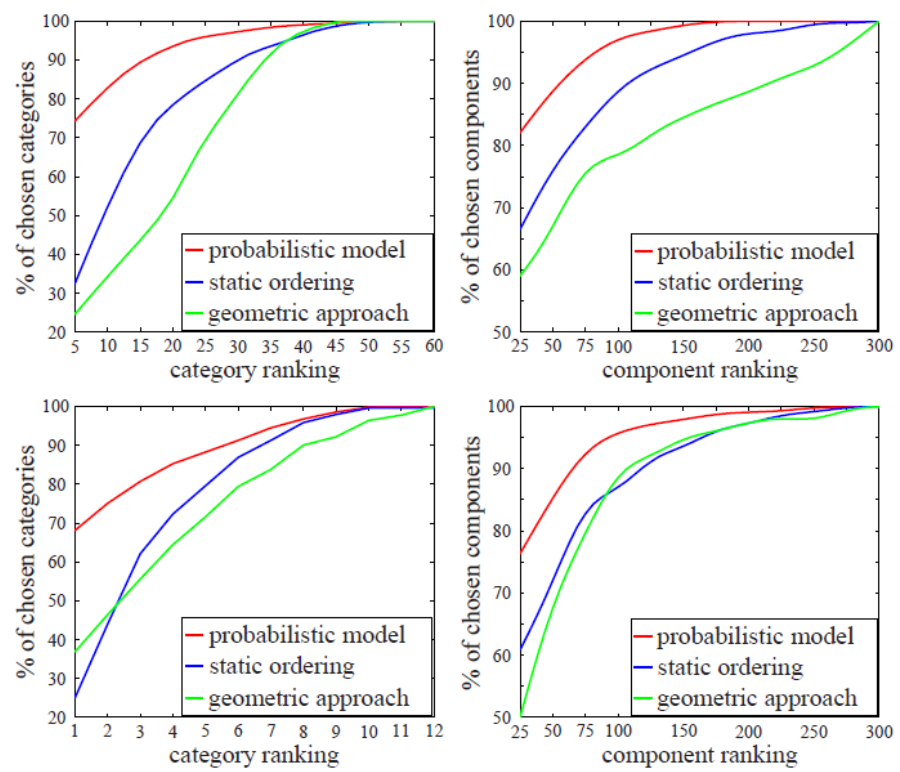

Figure 8.The Numerical Experiment Result for the Model 


\section{Conclusion and Summary}

Animation industry as a sunrise industry in the 21st century, in our country has developed rapidly in recent years, however, as the forefront of the whole animation industry and the development direction of 3D animation encountered many problems in its development process. Among them, the modelling of long production period and high cost of design, such as lack of innovative become outstanding problems. In recent years, experts use the evolutionary computation method for the study of a large number of innovative designs. In this paper, we conduct research on novel 3D modelling methodology for animation design based on computer graphics theory and mathematical analysis with Kinect implementation. We firstly introduce the basic concepts and application of Kinect system which serves as the preliminary of our research. Then, we conduct mathematical modelling for our system with theoretical analysis. The experimental simulation shows the effectiveness and correctness of our proposed approach compared with other popular methods. In the future, we schedule to conduct more related research to polish and modify the current approach under the guidance of the related literatures [16-25].

\section{References}

[1] R. Holly, Y. Lockerman, L. Cartwright and D, Pitera, "Experiments with a low-cost system for computer graphics material model acquisition", In IS\&T/SPIE Electronic Imaging, International Society for Optics and Photonics, (2015), pp. 939806-939806.

[2] Ehri L. C., "Orthographic mapping in the acquisition of sight word reading, spelling memory, and vocabulary learning", Scientific Studies of Reading, vol. 18, no. 1, (2014), pp. 5-21.

[3] A. Ken, J. P. Lewis and F. Pighin, "Scattered data interpolation for computer graphics", In ACM SIGGRAPH 2014 Courses, ACM, (2014), pp. 27.

[4] Q. Lijun, Z. Xiong and G. Zhou, "Image design of computer graphics and visual communication design and implementation", In Advanced Research and Technology in Industry Applications (WARTIA), 2014 IEEE Workshop on, IEEE, (2014), pp. 141-143.

[5] K. Upulee, J. M. Bieman and A. B. Hur, "Predicting Metamorphic Relations for Testing Scientific Software: A Machine Learning Approach Using Graph Kernels", (2014).

[6] B. Jan, K. Erleben and J. Trinkle, "Interactive simulation of rigid body dynamics in computer graphics", In Computer Graphics Forum, vol. 33, no. 1, (2014), pp. 246-270.

[7] L. Danielle, D. Espy, E. Fox, S. Pradhan and J. E. Deutsch, "Kinect-ing” With Clinicians: A Knowledge Translation Resource to Support Decision Making About Video Game Use in Rehabilitation", Physical therapy, (2014).

[8] Gray A. D., Jeff M. M., Erik E. S., Michael C. B., M. Skubic and S. L. Sherman, "Validation of the Microsoft Kinect as a Portable and Inexpensive Screening Tool for Identifying ACL Injury Risk", Orthopaedic Journal of Sports Medicine, 2325967114S00106, vol. 2, no. 2, (2014).

[9] G. Brook, G. Barry, D. Jackson, D. Mhiripiri, P. Olivier and L. Rochester, "Accuracy of the Microsoft Kinect sensor for measuring movement in people with Parkinson's disease", Gait \& posture, vol. 39, no. 4, (2014), pp. 1062-1068.

[10] Peng, Q. I. U., N. I. Chen, Z. H. A. N. G. Junzhong and C. A. O. Hui, "Research of Virtual Simulation of Traditional Chinese Bone Setting Techniques Based on Kinect Skeletal Information", Journal of Shandong University of Traditional Chinese Medicine, vol. 3, (2014), pp. 004.

[11] F. Joshua, T. Young, J. C. Peyton Jones and G. M. Clayton, "Integrating the Microsoft Kinect with Simulink: Real-time object tracking example." Mechatronics, IEEE/ASME Transactions on, vol. 19, no. 1, (2014), pp. 249-257.

[12] P. Frédéric, B. Dorronsoro, P. Bouvry and S. U. Khan, "It's not a bug, it's a feature: Wait-free asynchronous cellular genetic algorithm", In Parallel Processing and Applied Mathematics, Springer Berlin Heidelberg, (2014), pp. 361-370.

[13] Jayaraj V., J. J. Amalraj and S. Hemalatha, "An Analysis of Genetic Algorithm and Tabu Search Algorithm for Channel Optimization in Cognitive AdHoc Networks", (2014).

[14] L. Yan, Y. Feng and R. G. Pontius, "Spatially-explicit simulation of urban growth through self-adaptive genetic algorithm and cellular automata modeling", Land, vol. 3, no. 3, (2014), pp. 719-738.

[15] Parija S. R., P. K. Sahu and S. S. Singh, "Soft computing technique for cost reduction in cellular network", In Advances in Computing, Communications and Informatics (ICACCI, 2014 International Conference on, IEEE, (2014), pp. 890-894.

[16] Guo Y. D., "Three Dimensional Animation Design and Production Based on Computer Graphics", Advanced Materials Research, (2014), pp. 926-930. 
[17] Sbert M., Feixas M. and Rigau J., "Information theory tools for computer graphics", Synthesis Lectures on Computer Graphics \& Animation, no. 1, (2009).

[18] Huang X., Lin Y., Song M., Bu J. and Chun A. C., "3d facial expression synthesis based on nonlinear co-learning”, Journal of Computer-Aided Design \& Computer Graphics, vol. 23, no. 2, (2011), pp. 363362.

[19] Luciani A., Allaoui A. and Castagné N., "MORPHO-Map - A New Way to Model Animation of Topological Transformations", International Joint Conference on Computer Vision Imaging \& Computer Graphics Theory \& Applications, (2014).

[20] W. Haoxiang, and J. Wang, "An Effective Image Representation Method using Kernel Classification", Tools with Artificial Intelligence, ICTAI 2014. 26th IEEE International Conference on, (2014).

[21] Zhao Z. and Singer A., "Rotationally invariant image representation for viewing direction classification in cryo-EM", Journal of Structural Biology, vol. 186, no. 1, (2014), pp. 153-166.

[22] Hong Y., Si Z., Hu W., Zhu S. C. and Wu Y., "Unsupervised learning of compositional sparse code for natural image representation", Quarterly of Applied Mathematics, vol. 72, no. 2, (2014).

[23] Hu B. Q., Huang X. D., Zhou R. G., Wei Y. Y., Wan Q. and Pang C. Y., "A theoretical framework for quantum image representation and data loading scheme", Science China Information Sciences, vol. 57, no. 3, (2014), pp. 1-11.

[24] Yang X., Wu J., Zhang X., Jiang S. and Li K., "Destination image representation on the web: research on zhangjiajie travel related websites", Journal of the Graduates Sun Yat-Sen University (Natural Sciences、Medicine), (2014).

[25] Wei H., Lang B. and Zuo Q. S., "An image representation of infrastructure based on non-classical receptive field”, Soft Computing, vol. 18, no. 1, (2014), pp. 109-123. 
International Journal of Multimedia and Ubiquitous Engineering

Vol.10, No.10 (2015) 